\title{
Entanglement-free witnessing of quantum incompatibility in a high-dimensional system
}

\author{
Dian Wu $\odot,{ }^{1,2}$ Qi Zhao, ${ }^{1,2, *}$ Yi-Han Luo, ${ }^{1,2}$ Han-Sen Zhong, ${ }^{1,2}$ Li-Chao Peng $\odot,{ }^{1,2}$ Kai Chen $\odot,{ }^{1,2, \dagger}$ Peng Xue, ${ }^{3}$ Li Li, ${ }^{1,2}$ \\ Nai-Le Liu, ${ }^{1,2}$ Chao-Yang Lu ${ }^{1,1,2}$ and Jian-Wei Pan ${ }^{1,2}$ \\ ${ }^{1}$ Department of Modern Physics and National Laboratory for Physical Sciences at Microscale, Shanghai Branch, University of Science and \\ Technology of China, Hefei, Anhui 230026, China \\ ${ }^{2}$ CAS Center for Excellence and Synergetic Innovation Center in Quantum Information and Quantum Physics, Shanghai Branch, University \\ of Science and Technology of China, Hefei, Anhui 230026, China \\ ${ }^{3}$ Beijing Computational Science Research Center, Beijing 100084, China
}

(Received 13 June 2020; revised 12 February 2021; accepted 13 February 2021; published 6 April 2021)

\begin{abstract}
Quantum incompatibility, as an important quantum resource, is believed to play a key role in quantum information processing tasks. In order to detect this property, an entanglement-free incompatibility witness is proposed via constructing a combined quantum state discrimination task with prior-measurement and postmeasurement information strategies. Here we experimentally implement the witness of the incompatibility of mutually unbiased measurements under different noise levels in a qutrit system. Moreover, we estimate the robustness of incompatibility using collected data. Our work paves the path for understanding quantum incompatibility and exploring the features of the quantum resource.
\end{abstract}

DOI: 10.1103/PhysRevResearch.3.023017

\section{INTRODUCTION}

In quantum physics, some observables or measurements cannot commute with each other and cannot be performed simultaneously, which is quite different from its counterpart, classical physics [1,2]. This crucial quantum nonclassical feature is referred to as quantum incompatibility, which can be viewed as a quantum resource. This resource is shown to be essential for interesting quantum phenomena and quantum information processing tasks, including quantum nonlocality [3], quantum steering [4-6], uncertain relation [1], contextuality [7-10], and quantum key distribution [11]. For example, only incompatible measurements can lead to the violation of a Bell inequality [3] and every set of incompatible measurements can be used for quantum steering [4-6]. Thus it is fundamentally and practically significant to witness and quantify quantum incompatible measurements as a resource for achieving quantum advantages.

These applications motivate the attempt to investigating the detection methods of incompatibility. Some of the known detection tools are entanglement-based schemes, i.e., Bell nonlocal inequalities [12,13] and Einstein-Podolsky-Rosen steering schemes [4-6]. They usually require a quantum entanglement source and spacelike separation, which makes them experimentally challenging and not compact. Fortu-

\footnotetext{
*Corresponding author: zhaoqithu10@gmail.com

${ }^{\dagger}$ kaichen@ustc.edu.cn

Published by the American Physical Society under the terms of the Creative Commons Attribution 4.0 International license. Further distribution of this work must maintain attribution to the author(s) and the published article's title, journal citation, and DOI.
}

nately, entanglement is shown to be not necessarily needed for revealing the incompatibility features, which provides an alternative for witnessing quantum incompatibility [14-16]. Witnesses are tools widely used for detecting quantum resources, e.g, entanglement [17,18] and coherence [19-21], generally utilizing the linear combination of experimentally assessable observables.

Benefiting from the incompatible advantage in quantum states discrimination (QSD) tasks [14-16], Ref. [14] proposes an entanglement-free incompatibility witness via combining two disjoint state ensembles. The preparer can choose to reveal the chosen ensemble information before or after the measurement. For a set of incompatible measurements, the strategy with prior information can have a higher guessing probability than the post-measurement information strategy. And the gap between the guessing probabilities with these two strategies can exactly quantify the robustness of incompatibility in an optimal QSD [15,16,22]. These results provide both a direct testable method for incompatible measurements and operational meaning of quantum incompatibility. Moreover, with the rapid development of resource theory, other important quantum resources, e.g. magic, entanglement, and coherence can be detected via QSD [23,24]. There is also a quantitative connection between incompatibility, QSD, quantum coherence, entropic uncertainty relations, and accessible information $[23,25]$. Though there exists a lot of novel progress in the theoretical study of incompatibility, an implementation of the detection and quantitative estimation of this important resource is still left open, based on neither QSD task nor entanglement schemes. The exploration of incompatibility and QSD both can help deepen the understanding of quantum advantage and other quantum properties.

In this work, we report the direct test and quantitative confirmation of incompatible measurements. We implement an 
incompatibility witness of three-dimension mutually unbiased bases (MUB) measurements under different noise levels in a photonic quantum system. MUB measurements are among of the most representative incompatible measurements [26,27]. In quantum science, many quantum information protocols rely on the use of MUB, e.g., high dimensional entanglement detection [28], quantum state estimation [29], quantum secret sharing [30], and quantum key distribution [31]. In our demonstration, we implement three different witnesses to detect the incompatibility of MUB measurements under various noise regions, constructed from three different QSD tasks with prior-measurement and post-measurement information. Moreover, via the collected data, we give a lower bound of the robustness of incompatibility. To the best of our knowledge, our implementation is the first experimental report on the detection of incompatible measurements and quantitative estimation of the robustness of incompatibility. Our results show the feasibility of practical incompatibility witness in high-dimensional system, which provide a useful toolbox for the future study of incompatibility and MUB, and yield a significant step forward in the experimental quest for the practical detection of incompatibility.

\section{DETECTING QUANTUM INCOMPATIBILITY VIA QUANTUM STATE DISCRIMINATION TASKS}

Consider a pair of positive operator valued measurements (POVMs) $(M, N)$ with $M=\left\{M_{x}\right\}$ and $N=\left\{N_{y}\right\}$ satisfying $\sum_{x} M_{x}=\mathbb{I}, \sum_{y} N_{y}=\mathbb{I}$ with results $x$ and $y$, respectively. $M$ and $N$ are compatible if there exists a joint POVM measurement $J_{x, y}$ satisfying $\sum_{y} J_{x, y}=M_{x}, \sum_{x} J_{x, y}=N_{y}$. Recently, Ref. [14] showed that incompatible measurements can always be detected by a modified QSD task. A conventional QSD task involves an ensemble of quantum states $\mathcal{E}=\left\{p(z), \psi_{z}\right\}_{z}$ and a certain measurement $\left\{J_{z}\right\}$ used to guess the prepared states. The guessing probability for this ensemble is $P_{\text {guess }}(\mathcal{E}, J)=$ $\sum_{z} p(z) \operatorname{tr}\left(J_{z} \psi_{z}\right)$. The modified QSD involves a merged ensemble $\mathcal{E}$ constructed from two different subensembles $\mathcal{E}_{X}=$ $\left\{p(x), \psi_{x}\right\}_{x}, \mathcal{E}_{Y}=\left\{p(y), \phi_{y}\right\}_{y}$ with a proportion parameter $q$ as follows:

$$
\mathcal{E}=q \mathcal{E}_{X}+(1-q) \mathcal{E}_{Y}=\left\{p(x) q, \psi_{x} ; p(y)(1-q), \phi_{y}\right\}_{x, y} .
$$

The guesser, Bob has two strategies to guess the prepared states from the ensemble $\mathcal{E}$. In each round, if the information of set $X$ or $Y$ is announced before the measurement and the guesser uses measurements $M$ and $N$ to predicate the states from $\mathcal{E}_{X}$ and $\mathcal{E}_{Y}$, respectively, then the guessing probability with prior information is

$$
P_{\text {guess }}^{\text {prior }}(\mathcal{E}, M, N)=q P_{\text {guess }}\left(\mathcal{E}_{X}, M\right)+(1-q) P_{\text {guess }}\left(\mathcal{E}_{Y}, N\right) .
$$

In contrast, if the information of the subensemble is not revealed before the measurement, Bob has to use a joint measurement regardless of $\mathcal{E}_{X}$ or $\mathcal{E}_{Y}$, and the best guessing probability for Bob is $P_{\text {guess }}^{\text {post }}(\mathcal{E})=\max _{J} P_{\text {guess }}(\mathcal{E}, J)$ with a joint measurement $J$. A pair of compatible measurements can be simulated by a joint measurement, and the prior information would not affect the guessing probability. In contrast, incompatible measurements $M$ and $N$ show an advantage in a prior guessing probability. The incompatibility witness for measurements $M$ and $N$ can be constructed by this state ensemble $\mathcal{E}$,

$$
W=P_{\text {guess }}^{\text {post }}(\mathcal{E})-P_{\text {guess }}^{\text {prior }}(\mathcal{E}, M, N) .
$$

A negative value of witness implies the incompatibility of measurements $M$ and $N$. For any incompatible measurements, there always exists a witness or a combined state ensemble to detect their incompatibility. Moreover, according to [15], the robustness of incompatibility can be quantified by the ratio between two different strategies. The ensembles generated in our demonstration can serve as a lower bound of the robustness of incompatibility,

$$
1+\mathcal{R}(M, N) \geqslant \frac{P_{\text {guess }}^{\text {prior }}(\mathcal{E}, M, N)}{P_{\text {guess }}^{\text {post }}(\mathcal{E})},
$$

where the robustness of incompatibility $\mathcal{R}(M, N)$ is a resource monotone quantifying the minimal amount of noise added to measurements $M, N$ such that the mixture becomes compatible. The concrete expression of this quantifier and the related derivation of this lower bound are shown in Appendix A 2.

In this work, we focus on exploring the incompatibility of MUB measurements under different noise levels in a three-dimensional qutrit system. There are four MUB in qutrit system and we denote them as $\left\{\psi_{i}^{A}\right\}_{i \in\{1,2,3\}},\left\{\psi_{i}^{B}\right\}_{i \in\{1,2,3\}},\left\{\psi_{i}^{C}\right\}_{i \in\{1,2,3\}}, \quad$ and $\left\{\psi_{i}^{D}\right\}_{i \in\{1,2,3\}}$, which are shown in detail in Appendix A 1. We explore the incompatibility of $(A, B)$ and $(C, D)$ with noises. Taking the measurements $(A, B)$ as an example, we prepare the ensembles $\mathcal{E}_{X}$ and $\mathcal{E}_{Y}$,

$$
\begin{aligned}
& \mathcal{E}_{X}:\left\{\frac{1}{3}, r_{x}\left|\psi_{i}^{A}\right\rangle\left\langle\psi_{i}^{A}\right|+\left(1-r_{x}\right) \mathbb{I} / 3\right\}_{i}, \\
& \mathcal{E}_{Y}:\left\{\frac{1}{3}, r_{y}\left|\psi_{i}^{B}\right\rangle\left\langle\psi_{i}^{B}\right|+\left(1-r_{y}\right) \mathbb{I} / 3\right\}_{i},
\end{aligned}
$$

and merge them into a new ensemble via $\mathcal{E}=\frac{1}{2} \mathcal{E}_{X}+\frac{1}{2} \mathcal{E}_{Y}$. Different parameters $r_{x}$ and $r_{y}$ represent different state ensembles. We explore POVM measurements $(M, N)$ which are MUB measurements $(A, B)$ with noise parameters $s_{x}$ and $s_{y}$,

$$
\begin{aligned}
& M:\left\{s_{x}\left|\psi_{i}^{A}\right\rangle\left\langle\psi_{i}^{A}\right|+\left(1-s_{x}\right) \mathbb{I} / 3\right\}_{i}, \\
& N:\left\{s_{y}\left|\psi_{j}^{B}\right\rangle\left\langle\psi_{j}^{B}\right|+\left(1-s_{y}\right) \mathbb{I} / 3\right\}_{j} .
\end{aligned}
$$

In order to witness the incompatibility of $(M, N)$, we demonstrate QSD tasks with two strategies for the guesser. With the subensemble information $X$ or $Y$, we implement $M$ or $N$ according to it and the guessing probability is $P_{\text {guess }}^{\text {prior }}(\mathcal{E}, M, N)=$ $\frac{1}{2} P_{\text {guess }}\left(\mathcal{E}_{X}, M\right)+\frac{1}{2} P_{\text {guess }}\left(\mathcal{E}_{Y}, N\right)$. In the second strategy, we perform a fixed measurement regardless of the subset $X$ or $Y, P_{\text {guess }}^{\text {post }}(\mathcal{E})=P_{\text {guess }}(\mathcal{E}, J)$. The optimal measurements $J$ for different ensembles are shown in Appendix A 1.

\section{EXPERIMENTAL SETUP AND RESULTS}

In the experiment, we first implement the quantum incompatible witness. Our experimental setup is shown in Fig. 1. The left region in Fig. 1 shows the ensemble preparation setup. A femtosecond pulsed laser beam (394 nm, $76 \mathrm{MHz}$ ) is split into two parts to simultaneously create one photon pair by beamlike type-II spontaneous parametric down-conversion 


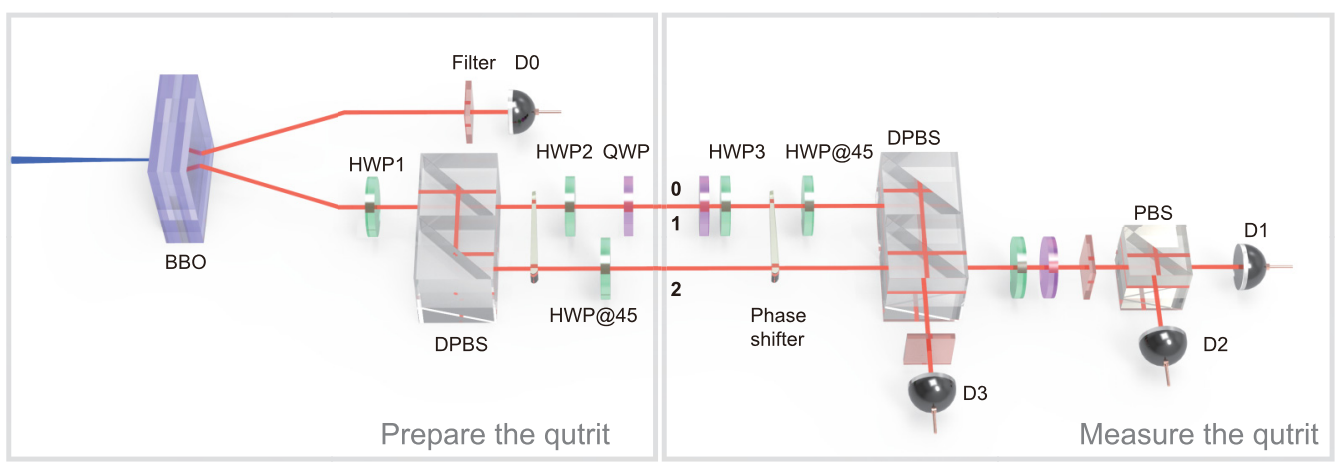

FIG. 1. Experimental setup for quantum incompatible witness. An ultraviolet pulsed laser is used to generate high-quality path-polarization qutrit states in a nonlinear crystal (BBO). The trigger photon goes through two 3-nm filters and one 8-nm filter for the extraordinary and ordinary photon, respectively. The other photon used for state preparation is sent through a half-wave plate (HWP1) which is set at $17.63^{\circ}$ and a double polarization beam splitter (DPBS). By changing two HWPs, a quarter-wave plate (QWP), and a phase shifter, twelve MUB states are generated. In the portion of measure the qutrit, DPBS, PBS, HWPs, and QWPs are used to construct the measurement observables. The angles of HWPs and QWPs are chosen to project the state to the eigenstates of the corresponding guessing measurement. The outputs are detected by four silicon avalanche photodiode detectors $D_{0}, D_{1}, D_{2}, D_{3}$.

from a BBO crystal. One photon serves as a coincidence trigger and the other photon after passing through one half-wave plate (HWP) and the first double polarization beam splitter (DPBS) is split into horizontal $(H)$ and vertical $(V)$ polarization modes on upper path and lower paths to $\sqrt{2 / 3}\left|H_{1}\right\rangle$, $\sqrt{1 / 3}\left|V_{2}\right\rangle$. In our spatial and polarizing hybrid qutrit state, we encode the polarization state of the single photon in the upper path as $|0\rangle,|1\rangle$ and the lower path $|V\rangle$ as $|2\rangle$. Applying a phase shifter, two half-wave plates, and one quarter-wave plate to adjust the phase among three paths $0,1,2$, we prepare twelve qutrit MUB eigenstates $\left\{\psi_{i}^{j}\right\}_{i \in\{1,2,3\}}(j=A, B, C, D)$. Note that here we cannot use coherent light to replace the single photon source, because it cannot exactly generate the required single party qutrit states in QSD tasks. We test the fidelity of the eigenstates in bases $A, B, C$, and $D$, $\left\{\psi_{i}^{j}\right\}_{i \in\{1,2,3\}}(j=A, B, C, D)$, shown in Table III, and the average fidelity of these prepared states in our experiment is $F=0.977$. The efficiency of a silicon single photon detector is about 0.7 , and the two-photon coincide count rate is $4 \mathrm{w} / \mathrm{s}$. By modifying the probabilities of sending different states, we prepare different ensembles as in Eq. (5) with $\left(r_{x}, r_{y}\right)=$ $(1,1),(0.5,1),(0.5,0.5)$. We denote these ensembles as $\mathcal{E}_{1}$, $\mathcal{E}_{2}, \mathcal{E}_{3}$ and the corresponding witnesses as $W_{1}, W_{2}, W_{3}$. Ensembles $\mathcal{E}_{4}, \mathcal{E}_{5}, \mathcal{E}_{6}$ and witnesses $W_{4}, W_{5}, W_{6}$ are for $\mathrm{C}, \mathrm{D}$ bases. We show the further details of prepared ensembles in Appendix A 1.

The right region in Fig. 1 shows the measurement setup. Here we combine different paths on the second double PBS. Fine adjustments of the delays between the different paths and two double PBSs constitute the phase-stable interferometer. During this process, the state in the upper path is integrated with the one in the lower path by the second double PBS. We use phase shift, two QWPs, two HWPs, and a PBS to perform different projections onto the qutrit states. In the post-measurement strategy, for three different ensembles, we perform the optimal compatible measurement $J^{k}=\left\{J_{i, j}^{k}\right\}_{i, j=1,2,3} \quad(k=1,2,3)$ from Ref. [14], each with nine POVM elements which are all projectors. We perform these projectors via modifying the angles of HWPs and QWPs, which are detailed in Appendix B 2. The guesser uses a fixed measurement at each round but he can postprocess the obtained measurement outcomes according to $\left(s_{x}, s_{y}\right)$. Consequently, we perform a total of $1.1 \times 10^{6}$ trials of QSD tasks with post-measurement information and we obtain the guessing probabilities with three different ensembles $P_{\text {guess }}^{\text {post }}\left(\mathcal{E}_{k}, J^{k}\right)=0.7700 \pm$ $0.0009,0.6805 \pm 0.0006,0.5523 \pm 0.0004(k=1,2,3)$ for $A, B$ bases and $P_{\text {guess }}^{\text {post }}\left(\mathcal{E}_{k}, J^{k}\right)=0.7684 \pm 0.0009,0.6794 \pm$ $0.0006,0.5507 \pm 0.0005(k=4,5,6)$ for $C, D$ bases.

In the other strategy with the prior-measurement information, the guesser has access to prior information of $X$ and $Y$. Then, he can choose a measurement $M$ for the state from $\mathcal{E}_{X}$ and a measurement $N$ for $\mathcal{E}_{Y}$ in Eq. (6). In our demonstration, we faithfully perform the projection measurement $A, B(C, D)$ with $s_{x}=1, s_{y}=1$ in Eq. (6) and obtain the general measurement results for $M$ and $N$ via post-processing,

$$
\begin{aligned}
P_{\text {guess }}^{\text {prior }}\left(\mathcal{E}_{i}, M, N\right) & =\frac{1}{2}\left[P_{\text {guess }}^{\text {prior }}\left(\mathcal{E}_{X}^{i}, M\right)+P_{\text {guess }}^{\text {prior }}\left(\mathcal{E}_{Y}^{i}, N\right)\right], \\
P_{\text {guess }}^{\text {prior }}\left(\mathcal{E}_{X}^{i}, M\right) & =s_{X} P_{\text {guess }}^{\text {prior }}\left(\mathcal{E}_{X}^{i}, A\right)+\left(1-s_{x}\right) / 3, \\
P_{\text {guess }}^{\text {prior }}\left(\mathcal{E}_{Y}^{i}, N\right) & =s_{y} P_{\text {guess }}^{\text {prior }}\left(\mathcal{E}_{Y}^{i}, B\right)+\left(1-s_{y}\right) / 3 .
\end{aligned}
$$
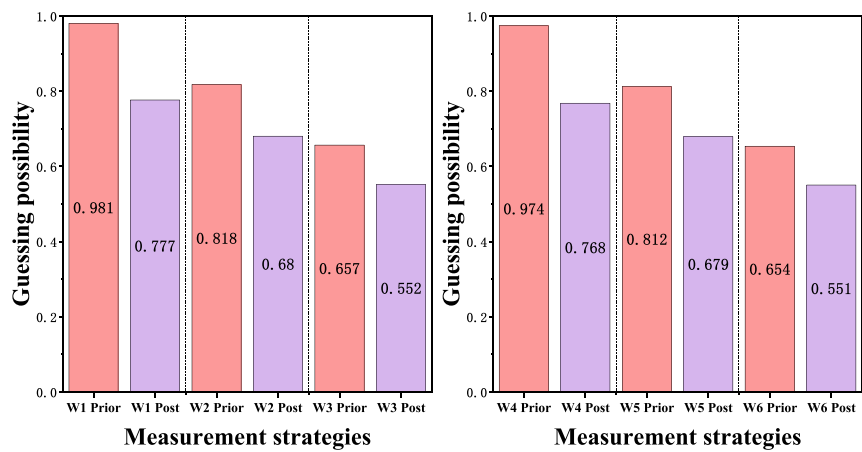

FIG. 2. Experimental guessing probabilities with prior- and postmeasurement information $P_{\text {guess }}^{\text {prior }}, P_{\text {guess }}^{\text {post }}$ in various witnesses. 
TABLE I. Prior and post guessing probabilities with six different ensembles.

\begin{tabular}{|c|c|c|c|c|c|}
\hline \multicolumn{6}{|c|}{$\begin{array}{c}\mathrm{A} \text { and } \mathrm{B} \text { bases } \\
\text { Ensembles } \mathcal{E}_{i},\left(r_{x}, r_{y}\right)\end{array}$} \\
\hline \multicolumn{2}{|c|}{$\mathcal{E}_{1},(1,1)$} & \multicolumn{2}{|c|}{$\begin{array}{c}\mathcal{E}_{2},(0.5,1) \\
\text { Guessing probabilities }\end{array}$} & \multicolumn{2}{|c|}{$\mathcal{E}_{3},(0.5,0.5)$} \\
\hline $\begin{array}{l}P_{\text {guess }}^{\text {prior }} \\
0.9806\end{array}$ & $\begin{array}{c}P_{\text {guess }}^{\text {post }} \\
0.7700\end{array}$ & $\begin{array}{c}P_{\text {guess }}^{\text {prior }} \\
0.8177\end{array}$ & $\begin{array}{c}P_{\text {guess }}^{\text {post }} \\
0.6805\end{array}$ & $\begin{array}{c}P_{\text {guess }}^{\text {prior }} \\
0.6570\end{array}$ & $\begin{array}{c}P_{\text {guess }}^{\text {post }} \\
0.5523\end{array}$ \\
\hline \multicolumn{6}{|c|}{$\begin{array}{c}\mathrm{C} \text { and } \mathrm{D} \text { bases } \\
\text { Ensembles } \mathcal{E}_{i},\left(r_{x}, r_{y}\right)\end{array}$} \\
\hline \multicolumn{2}{|c|}{$\mathcal{E}_{4},(1,1)$} & \multicolumn{2}{|c|}{$\begin{array}{c}\mathcal{E}_{5},(0.5,1) \\
\text { Guessing probabilities }\end{array}$} & \multicolumn{2}{|c|}{$\mathcal{E}_{6},(0.5,0.5)$} \\
\hline $\begin{array}{l}P_{\text {guess }}^{\text {prior }} \\
0.9742\end{array}$ & $\begin{array}{c}P_{\text {guess }}^{\text {post }} \\
0.7684\end{array}$ & $\begin{array}{c}P_{\text {guess }}^{\text {prior }} \\
0.8123\end{array}$ & $\begin{array}{c}P_{\text {guess }}^{\text {post }} \\
0.6794\end{array}$ & $\begin{array}{c}P_{\text {guess }}^{\text {prior }} \\
0.6538\end{array}$ & $\begin{array}{c}P_{\text {guess }}^{\text {post }} \\
0.5507\end{array}$ \\
\hline
\end{tabular}

We perform a total $6.1 \times 10^{5}$ trials of QSD tasks in the priormeasurement information case, and obtain $P_{\text {guess }}^{\text {prior }}\left(\mathcal{E}_{k}, A, B\right)=$ $0.9806 \pm 0.0005,0.8177 \pm 0.0005,0.6570 \pm 0.0005(k=1$, $2,3)$ for $\mathrm{A}, \mathrm{B}$ bases, and $P_{\text {guess }}^{\text {prior }}\left(\mathcal{E}_{k}, C, D\right)=0.9742 \pm$ $0.0006,0.8123 \pm 0.0006,0.6538 \pm 0.0006(k=4,5,6)$ for $\mathrm{C}$, D bases. We show the comparison between guessing probabilities with prior- and post-measurement information in Fig. 2. We summarize all the ensemble settings and corresponding guessing probabilities in Table I.

According to Eq. (3), we can obtain the incompatible witness via comparing prior and post guessing probabilities,

$$
\begin{aligned}
W_{i}= & P_{\text {guess }}^{\text {post }}\left(\mathcal{E}_{i}\right)-P_{\text {guess }}^{\text {prior }}\left(\mathcal{E}_{i}, M, N\right) \\
= & P_{\text {guess }}^{\text {post }}\left(\mathcal{E}_{i}\right)-\frac{1}{2}\left[s_{x} P_{\text {guess }}^{\text {prior }}\left(\mathcal{E}_{X}^{i}, A\right)+s_{y} P_{\text {guess }}^{\text {prior }}\left(\mathcal{E}_{Y}^{i}, B\right)\right] \\
& -\frac{1}{6}\left(2-s_{x}-s_{y}\right) .
\end{aligned}
$$

We implement six different ensembles to demonstrate different witnesses. Though we experimentally obtain the post guessing probabilities, here we still use theoretical values to make the incompatibility detection rigorous. We show the incompatibility and compatibility regions in Fig. 3. Note that not all quantum state discrimination tasks are valid for detecting incompatibility, and each task has different detectable region. We show the detectable region of our witnesses, and $W_{1}, W_{3}$ ( $W_{4}$ and $W_{6}$ ) present the same witness curve in Fig. 3.

In Ref. [15], the robustness of incompatibility of $\mathcal{R}(M, N)$ can be characterized by quantum state discrimination task. Combining with the post-processing, our results can also serve as a lower bound of the robustness of incompatibility,

$$
\begin{aligned}
1+ & \mathcal{R}(M, N) \\
\geqslant & \geqslant \frac{s_{x} P_{\text {guess }}^{\text {prior }}\left(\mathcal{E}_{X}^{i}, A\right)+s_{y} P_{\text {guess }}^{\text {prior }}\left(\mathcal{E}_{Y}^{i}, B\right)+\left(2-s_{x}-s_{y}\right) / 3}{2 P_{\text {guess }}^{\text {post }}\left(\mathcal{E}_{i}\right)},
\end{aligned}
$$

where $\mathcal{E}_{i}$ are the ensembles generated in our experiment. Similarly, $A, B$ bases can be replaced with $C$ and $D$ bases. We show the lower bound of the robustness for incompatibility under different noise levels in Fig. 4. When $\left(s_{x}, s_{y}\right)=(1,1)$,

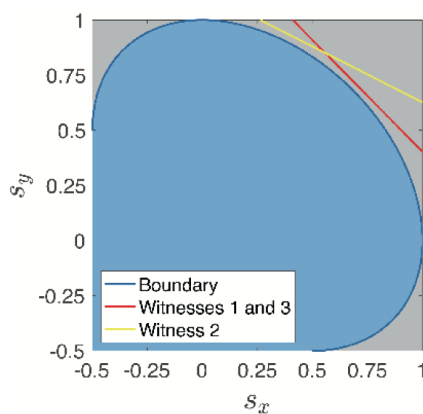

(a)

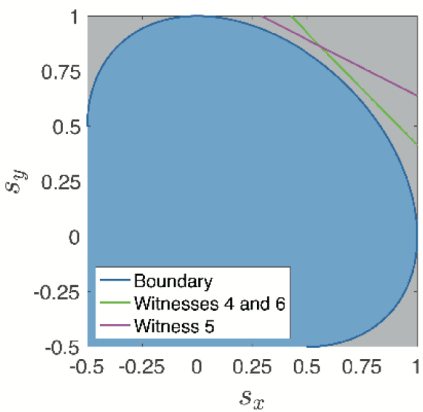

(b)
FIG. 3. Two measurements $(M, N)$ corresponding to the set of $\left(s_{X}, s_{y}\right)$ in Eq. (6). (a) Compatible and incompatible regions in $A$ and $B$ bases. (b) Compatible and incompatible regions in $C$ and $D$ bases. The measurements in the blue region and grey region are compatible and incompatible, respectively. The measurements above the curves in the top-right corner region are detected by our witnesses.

we obtain $\mathcal{R}(A, B)=0.2620, \mathcal{R}(C, D)=0.2679$. Note that from Fig. 4 the robustness of incompatibility of some incompatible measurements is zero, because the ensembles we implemented are not optimal for these measurements. Thus, from our implementation, we could only give a lower bound of this quantifier.

\section{SUMMARY}

The incompatibility witness without entanglement is an effective and realistic tool in the detection of incompatible properties. We have experimentally verified the incompatibility of MUB measurements under different noise levels, which shows the feasibility of this witness. Furthermore, we quantitatively benchmark the incompatible measurements by estimating the robustness of incompatibility. Our demonstration is based on quantum state discrimination (QSD) tasks, which is entanglement free and not based on the Bell inequality. Thus, similar to the conventional entanglement witness, we trust the state preparation and measurements devices.

Applying a similar scheme, our demonstration can also be extended to higher dimensional incompatible measurement detection. Similar results are also obtained for the detection of key properties in quantum evolution, for instance the incom-

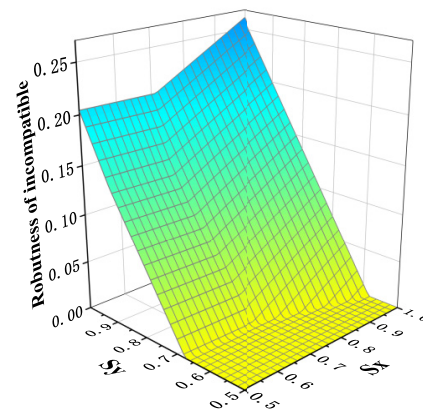

(a)

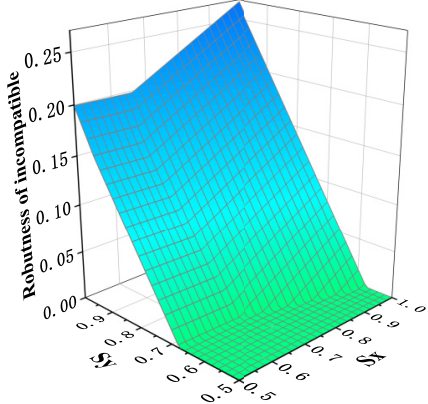

(b)
FIG. 4. The lower bound of the robustness of incompatibility versus noises $\left(s_{x}, s_{y}\right)$ in measurements $M$ and $N$. (a) The robustness of incompatibility in $A$ and $B$ bases. (b) The robustness of incompatibility in $C$ and $D$ bases. 
patibility of quantum channels and quantum memory [32-34]. Our demonstration paves the way for future implementation and applications for quantum resource detection and discrimination game schemes.

\section{ACKNOWLEDGMENTS}

This work was supported by the National Natural Science Foundation of China (91836303, 11674308, and 11525419), the Chinese Academy of Sciences, the National Fundamental Research Program (2018YFA0306100), and the Anhui Initiative in Quantum Information Technologies.

D.W. and Q.Z. contributed equally to this work.

\section{APPENDIX A: THEORETICAL DETAILS}

\section{MUB and optimal joint measurements}

In the three-dimensional system, we have four mutually unbiased bases (MUB). We choose these MUB as follows:

$A:\{|0\rangle,|1\rangle,|2\rangle\}$,

$B: \frac{1}{\sqrt{3}}\left\{|0\rangle+|1\rangle+|2\rangle,|0\rangle+\omega|1\rangle+\omega^{2}|2\rangle,|0\rangle+\omega^{2}|1\rangle+\omega|2\rangle\right\}$,

$C: \frac{1}{\sqrt{3}}\{\omega|0\rangle+|1\rangle+|2\rangle,|0\rangle+\omega|1\rangle+|2\rangle,|0\rangle+|1\rangle+\omega|2\rangle\}$,

$D: \frac{1}{\sqrt{3}}\left\{\omega^{2}|0\rangle+|1\rangle+|2\rangle,|0\rangle+\omega^{2}|1\rangle+|2\rangle,|0\rangle+|1\rangle+\omega^{2}|2\rangle\right\}$,

where $\omega=\exp (i 2 \pi / 3)$. We explore the incompatibility of bases $(A, B)$ and $(C, D)$ under different noises. Here we take bases $(A, B)$ as an example. In the modified QSD task $\mathcal{E}=$ $\frac{1}{2} \mathcal{E}_{X}+\frac{1}{2} \mathcal{E}_{Y}$, we prepare the ensembles $\mathcal{E}_{X}$ and $\mathcal{E}_{Y}$,

$$
\begin{aligned}
& \mathcal{E}_{X}:\left\{\frac{1}{3}, r_{x}\left|\psi_{i}^{A}\right\rangle\left\langle\psi_{i}^{A}\right|+\left(1-r_{x}\right) \mathbb{I} / 3\right\}_{i}, \\
& \mathcal{E}_{Y}:\left\{\frac{1}{3}, r_{y}\left|\psi_{i}^{B}\right\rangle\left\langle\psi_{i}^{B}\right|+\left(1-r_{y}\right) \mathbb{I} / 3\right\}_{i},
\end{aligned}
$$

where

$$
\left|\psi_{0}^{A}\right\rangle=|0\rangle,\left|\psi_{1}^{A}\right\rangle=|1\rangle,\left|\psi_{2}^{A}\right\rangle=|2\rangle
$$

$\left|\psi_{0}^{B}\right\rangle=\frac{1}{\sqrt{3}}(|0\rangle+|1\rangle+|2\rangle),\left|\psi_{1}^{B}\right\rangle=\frac{1}{\sqrt{3}}(|0\rangle+\omega|1\rangle+$ $\left.\omega^{2}|2\rangle\right),\left|\psi_{2}^{B}\right\rangle=\frac{1}{\sqrt{3}}\left(|0\rangle+\omega^{2}|1\rangle+\omega|2\rangle\right)$. Parameters $r_{x}$ and $r_{y}$ represent different states ensembles. The pair of POVM measurements $(M, N)$ are MUB measurements $(A, B)$ under different noise parameters $s_{x}$ and $s_{y}$,

$$
\begin{aligned}
& M:\left\{s_{x}\left|\psi_{i}^{A}\right\rangle\left\langle\psi_{i}^{A}\right|+\left(1-s_{x}\right) \mathbb{I} / 3\right\}_{i}, \\
& N:\left\{s_{y}\left|\psi_{j}^{B}\right\rangle\left\langle\psi_{j}^{B}\right|+\left(1-s_{y}\right) \mathbb{I} / 3\right\}_{j} .
\end{aligned}
$$

The incompatibility of $(M, N)$ can be witnessed by the difference between the guessing probability with two strategies in QSD tasks, $P_{\text {guess }}^{\text {prior }}(\mathcal{E}, M, N)=\frac{1}{2} P_{\text {guess }}\left(\mathcal{E}_{X}, M\right)+$ $\frac{1}{2} P_{\text {guess }}\left(\mathcal{E}_{Y}, N\right)$ and $P_{\text {guess }}^{\text {post }}(\mathcal{E})=P_{\text {guess }}(\mathcal{E}, J)$. The optimal joint measurements $J$ are given in Ref. [32]:

$$
\begin{aligned}
J_{i, j}= & b\left(r_{x}\left|\psi_{i}\right\rangle\left\langle\psi_{i}\left|+r_{y}\right| \phi_{j}\right\rangle\left\langle\phi_{j}\right|\right)-\frac{d c}{d-1}\left(\left|\psi_{i}\right\rangle\left\langle\psi_{i}|+| \phi_{j}\right\rangle\left\langle\phi_{j}\right|\right. \\
& \left.-\left|\psi_{i}\right\rangle\left\langle\psi_{i}|| \phi_{j}\right\rangle\left\langle\phi_{j}|-| \phi_{j}\right\rangle\left\langle\phi_{j}|| \psi_{i}\right\rangle\left\langle\psi_{i}\right|\right),
\end{aligned}
$$

where $b=\frac{1}{\sqrt{r_{x}^{2}+r_{y}^{2}-\frac{2}{3} r_{x} r_{y}}}, c=\frac{1}{2}\left(\frac{s_{x}+s_{y}}{\sqrt{r_{x}^{2}+r_{y}^{2}-\frac{2}{3} r_{x} r_{y}}}-1\right)$, and $d$ is the dimension. Note that these elements $J_{i, j}$ are all projectors. For $A, B$ bases, when $r_{x}, r_{y}=1$ and $r_{x}=1 / 2, r_{y}=1 / 2$, projectors $J_{i, j}$ are as follows. Here we write down the vectors as

$$
\begin{aligned}
& J_{1,1}=(0.8881,0.3251,0.3251)^{\mathrm{T}}, \\
& J_{1,2}=(0.8881,-0.1625-0.2815 i,-0.1625+0.2815 i)^{\mathrm{T}}, \\
& J_{1,3}=(0.8881,-0.1625+0.2815 i,-0.1625-0.2815 i)^{\mathrm{T}}, \\
& J_{2,1}=(0.3251,0.8881,0.3251)^{\mathrm{T}}, \\
& J_{2,2}=(-0.1625+0.2815 i, 0.8881,-0.1625-0.2815 i)^{\mathrm{T}}, \\
& J_{2,3}=(-0.1625-0.2815 i, 0.8881,-0.1625+0.2815 i)^{\mathrm{T}}, \\
& J_{3,1}=(0.3251,0.3251,0.8881)^{\mathrm{T}}, \\
& J_{3,2}=(-0.1625-0.2815 i,-0.1625+0.2815 i, 0.8881)^{\mathrm{T}}, \\
& J_{3,3}=(-0.1625+0.2815 i,-0.1625-0.2815 i, 0.8881)^{\mathrm{T}} .
\end{aligned}
$$

When $r_{x}=1 / 2, r_{y}=1$, vectors $J_{i, j}$ are as follows:

$$
\begin{aligned}
& J_{1,1}=(0.7662,0.4544,0.4544)^{\mathrm{T}}, \\
& J_{1,2}=(0.7662,-0.2272-0.3935 i,-0.2272+0.3935 i)^{\mathrm{T}}, \\
& J_{1,3}=(0.7662,-0.2272+0.3935 i,-0.2272-0.3935 i)^{\mathrm{T}}, \\
& J_{2,1}=(0.4544,0.7662,0.4544)^{\mathrm{T}}, \\
& J_{2,2}=(-0.2272+0.3935 i, 0.7662,-0.2272-0.3935 i)^{\mathrm{T}}, \\
& J_{2,3}=(-0.2272-0.3935 i, 0.7662,-0.2272+0.3935 i)^{\mathrm{T}}, \\
& J_{3,1}=(0.4544,0.4544,0.7662)^{\mathrm{T}}, \\
& J_{3,2}=(-0.2272-0.3935 i,-0.2272+0.3935 i, 0.7662)^{\mathrm{T}}, \\
& J_{3,3}=(-0.2272+0.3935 i,-0.2272-0.3935 i, 0.7662)^{\mathrm{T}} .
\end{aligned}
$$

For $C$ and $D$ bases, when $r_{x}=r_{y}=1$ and $r_{x}=r_{y}=1 / 2$, $J_{i, j}$ are

$$
\begin{aligned}
& J_{1,1}=(-0.4597,0.6280,0.6280)^{\mathrm{T}}, \\
& J_{1,2}=(-0.3140-0.5438 i, 0.6280,0.2299-0.3981 i)^{\mathrm{T}}, \\
& J_{1,3}=(0.6280,0.2299-0.3981 i,-0.3140-0.5438 i)^{\mathrm{T}},
\end{aligned}
$$

TABLE II. State preparation angles.

\begin{tabular}{lccc}
\hline \hline State & Phase shifter & HWP2 & QWP \\
\hline$B 1$ & 0 & 0 & 45 \\
$B 2$ & 0 & -60 & 45 \\
$B 3$ & 0 & 60 & 45 \\
$C 1$ & 240 & 60 & 45 \\
$C 2$ & 240 & -60 & 45 \\
$C 3$ & 120 & 0 & 45 \\
$D 1$ & 120 & -60 & 45 \\
$D 2$ & 120 & 60 & 45 \\
$D 3$ & 240 & 0 & 45 \\
\hline \hline
\end{tabular}


TABLE III. Fidelity of prepared states.

\begin{tabular}{lccc}
\hline \hline Fidelity & $\left|\psi_{1}\right\rangle$ & $\left|\psi_{2}\right\rangle$ & $\left|\psi_{3}\right\rangle$ \\
\hline$A$ & 0.9735 & 0.9836 & 0.9979 \\
$B$ & 0.9793 & 0.9697 & 0.9795 \\
$C$ & 0.9738 & 0.9853 & 0.9831 \\
$D$ & 0.9626 & 0.9641 & 0.9762 \\
\hline \hline
\end{tabular}

$$
J_{2,1}=(0.6280,-0.3140-0.5438 i, 0.2299-0.3981 i)^{\mathrm{T}} \text {, }
$$$$
J_{2,2}=(0.6280,-0.4597,0.6280)^{\mathrm{T}} \text {, }
$$$$
J_{2,3}=(0.2299-0.3981 i,-0.3140-0.5438 i, 0.6280)^{\mathrm{T}} \text {, }
$$$$
J_{3,1}=(0.6280,0.2299-0.3981 i,-0.3140-0.5438 i)^{\mathrm{T}} \text {, }
$$$$
J_{3,2}=(0.2299-0.3981 i, 0.6280,-0.3140-0.5438 i)^{\mathrm{T}} \text {, }
$$$$
J_{3,3}=(0.6280,0.6280,-0.4597)^{\mathrm{T}} \text {. }
$$

When $r_{x}=1 / 2, r_{y}=1, J_{i, j}$ are

$$
\begin{aligned}
& J_{1,1}=(-0.4233+0.2444 i, 0.6169,0.6169)^{\mathrm{T}}, \\
& J_{1,2}=(-0.3084-0.5342 i, 0.6169,-0.4888 i)^{\mathrm{T}}, \\
& J_{1,3}=(0.6169,0.4233+0.2444 i,-0.3084+0.5342 i)^{\mathrm{T}}, \\
& J_{2,1}=(-0.3084+0.5342 i, 0.6169,0.4233+0.2444 i)^{\mathrm{T}}, \\
& J_{2,2}=(0.6169,-0.4233+0.2444 i, 0.6169)^{\mathrm{T}}, \\
& J_{2,3}=(0.4233+0.2444 i, 0.6169,-0.3084+0.5342 i)^{\mathrm{T}}, \\
& J_{3,1}=(0.6169,-0.4888 i,-0.3084-0.5342 i)^{\mathrm{T}}, \\
& J_{3,2}=(0.4233+0.2444 i,-0.3084+0.5342 i, 0.6169)^{\mathrm{T}}, \\
& J_{3,3}=(0.6169,0.6169,-0.4233+0.2444 i)^{\mathrm{T}} .
\end{aligned}
$$

\section{The robustness of incompatibility}

Consider a fixed pair of incompatible measurements $(M, N)$; the robustness of incompatibility (ROI) of the measurements $\mathcal{R}(M, N)$ is defined as the minimal amount of an arbitrary set of measurements which makes the mixture compatible:

$$
\mathcal{R}(M, N)=\min r
$$

TABLE IV. Projectors angles: $A$ and $B$ bases with $\left(r_{x}, r_{y}\right)=$ $(1,1),(1 / 2,1 / 2)$.

\begin{tabular}{lccccc}
\hline \hline Projector & Phase shifter & QWP & HWP3 & QWP & HWP5 \\
\hline$J_{1,1}$ & 0 & 20.2 & 10.1 & 71 & 35.5 \\
$J_{1,2}$ & 63.5 & 168.6 & 75.8 & 71 & 35.5 \\
$J_{1,3}$ & 116.5 & 168.6 & 2.8 & 71 & 35.5 \\
$J_{2,1}$ & 0 & 70 & 35 & 71 & 35.5 \\
$J_{2,2}$ & 26.4 & 11.5 & 59.2 & 71 & 35.5 \\
$J_{2,3}$ & 153.5 & 11.5 & 42.2 & 71 & 35.5 \\
$J_{3,1}$ & 0 & 45 & 22.5 & 27.4 & 13.7 \\
$J_{3,2}$ & 0 & 45 & 82.5 & 27.4 & 13.7 \\
$J_{3,3}$ & 270 & 135 & 82.5 & 27.4 & 13.7 \\
\hline \hline
\end{tabular}

TABLE V. Projectors angles: $A$ and $B$ bases with $\left(r_{x}, r_{y}\right)=(1 / 2,1)$.

\begin{tabular}{lccccc}
\hline \hline Projector & Phase shifter & QWP & HWP3 & QWP & HWP5 \\
\hline$J_{1,1}$ & 0 & 30.6 & 15.3 & 63 & 31.5 \\
$J_{1,2}$ & 160.1 & 68.8 & 1.8 & 63 & 31.5 \\
$J_{1,3}$ & 109.9 & 158.8 & 1.8 & 63 & 31.5 \\
$J_{2,1}$ & 270 & 149.4 & 29.7 & 63 & 31.5 \\
$J_{2,2}$ & 109.9 & 111.2 & 43.2 & 63 & 31.5 \\
$J_{2,3}$ & 160.1 & 21.2 & 43.2 & 63 & 31.5 \\
$J_{3,1}$ & 0 & 45 & 22.5 & 40 & 20 \\
$J_{3,2}$ & 90 & 135 & 52.5 & 40 & 20 \\
$J_{3,3}$ & 180 & 45 & 52.5 & 40 & 20 \\
\hline \hline
\end{tabular}

$$
\text { s.t. } \begin{aligned}
\frac{M_{x}+r P_{x}}{1+r} & =\sum_{\lambda} p(x \mid \lambda) G_{\lambda}, \forall x, \\
\frac{N_{y}+r Q_{y}}{1+r} & =\sum_{\lambda} q(y \mid \lambda) G_{\lambda}, \forall y, \\
P_{x} & \geqslant 0, \forall x, \sum_{x} P_{x}=\mathbb{I}, \\
Q_{y} & \geqslant 0, \forall y, \sum_{y} Q_{y}=\mathbb{I}, \\
\sum_{x} p(x \mid \lambda) & =1, \sum_{x} q(y \mid \lambda)=1, \\
G_{\lambda} & \geqslant 0, \sum_{\lambda} G_{\lambda}=\mathbb{I},
\end{aligned}
$$

where the minimization is over $r,\left(\{P\}_{x},\{Q\}_{y}\right),\left\{G_{\lambda}\right\}$, $\{p(x \mid \lambda)\}_{x, \lambda}$, and $\{q(y \mid \lambda)\}_{y, \lambda}$.

In Ref. [15], the robustness of incompatibility of $\mathcal{R}(M, N)$ can be characterized by a quantum state discrimination task,

$$
1+\mathcal{R}(M, N)=\max _{\mathcal{E}} \frac{P_{\text {guess }}^{\text {prior } *}(\mathcal{E}, M, N)}{P_{\text {guess }}^{\text {post }}(\mathcal{E})}
$$

where the definitions of $P_{\text {guess }}^{\text {prior* }}$ and $P_{\text {guess }}^{\text {post* }}$ are slightly different with $P_{\text {guess }}^{\text {prior }}$ and $P_{\text {guess }}^{\text {post }}$ in the main text. Consider the combined ensemble $\mathcal{E}=\frac{1}{2} \mathcal{E}_{x}+\frac{1}{2} \mathcal{E}_{y}$, then

$$
\begin{aligned}
P_{\text {guess }}^{\text {post } *}(\mathcal{E})= & \frac{1}{2} \max _{T} \sum_{x, y, v, a} p(v)\left[p(y) \operatorname{tr}\left(\psi_{y} G_{a, v}\right) \tilde{p}(y \mid a, v)\right. \\
& \left.+p(x) \operatorname{tr}\left(\psi_{x} G_{a, v}\right) \tilde{q}(x \mid a, v)\right] .
\end{aligned}
$$

where the maximization is over $T=$ $\left\{p(v),\{G\}_{a, v}, \tilde{p}(y \mid a, v), \tilde{q}(x \mid a, v)\right\}$. According to Ref. [15], this guessing probability with more general strategy can be rewritten into

$$
\begin{aligned}
& \frac{1}{2} \sum_{x, y, v, a} p(v)\left[p(y) \operatorname{tr}\left(\psi_{y} G_{a, v}\right) \tilde{p}(y \mid a, v)+p(x)\right. \\
& \left.\quad \times \operatorname{tr}\left(\psi_{x} G_{a, v}\right) \tilde{q}(x \mid a, v)\right] \\
& =\frac{1}{2} \sum_{x, y}\left[p(y) \operatorname{tr}\left(\psi_{y} J_{x, y}\right)+p(x) \operatorname{tr}\left(\psi_{x} J_{x, y}\right)\right]
\end{aligned}
$$


TABLE VI. Projectors angles: $C$ and $D$ bases with $\left(r_{x}, r_{y}\right)=$ $(1,1),(1 / 2,1 / 2)$.

\begin{tabular}{lccccc}
\hline \hline Projector & Phase shifter & QWP & HWP3 & QWP & HWP5 \\
\hline$J_{1,1}$ & 270 & 36.2 & 63.1 & 51.1 & 25.55 \\
$J_{1,2}$ & 90 & 135 & 82.5 & 62.6 & 31.3 \\
$J_{1,3}$ & 166.2 & 118.8 & 0.5 & 51.1 & 25.55 \\
$J_{2,1}$ & 180 & 45 & 82.5 & 62.6 & 31.3 \\
$J_{2,2}$ & 90 & 53.8 & 71.9 & 51.1 & 25.55 \\
$J_{2,3}$ & 166.2 & 151.2 & 44.5 & 51.1 & 25.55 \\
$J_{3,1}$ & 103.8 & 28.8 & 0.5 & 51.1 & 25.55 \\
$J_{3,2}$ & 103.8 & 61.2 & 44.5 & 51.1 & 25.55 \\
$J_{3,3}$ & 180 & 45 & 22.5 & 62.6 & 31.3 \\
\hline \hline
\end{tabular}

Thus $P_{\text {guess }}^{\text {post }}(\mathcal{E})$ is equivalent to the definition used in our main text, $P_{\text {guess }}^{\text {post }}(\mathcal{E})=\max _{J} \frac{1}{2} \sum_{x, y}\left[p(y) \operatorname{tr}\left(\psi_{y} J_{x, y}\right)+\right.$ $\left.p(x) \operatorname{tr}\left(\psi_{x} J_{x, y}\right)\right]$ :

$$
P_{\text {guess }}^{\text {post } *}(\mathcal{E})=P_{\text {guess }}^{\text {post }}(\mathcal{E}) .
$$

Consequently, we can use the ratio between $P_{\text {guess }}^{\text {prior }}\left(\mathcal{E}_{0}, M, N\right)$, $P_{\text {guess }}^{\text {post }}\left(\mathcal{E}_{0}\right)$ to lower bound the robustness of incompatibility,

$$
\begin{aligned}
1+\mathcal{R}(M, N) & =\max _{\mathcal{E}} \frac{P_{\text {guess }}^{\text {prior } *}(\mathcal{E}, M, N)}{P_{\text {guess }}^{\text {post } *}(\mathcal{E})} \geqslant \max _{\mathcal{E}} \frac{P_{\text {guess }}^{\text {prior }}(\mathcal{E}, M, N)}{P_{\text {guess }}^{\text {post }}(\mathcal{E})} \\
& \geqslant \frac{P_{\text {guess }}^{\text {prior }}\left(\mathcal{E}_{0}, M, N\right)}{P_{\text {guess }}^{\text {post }}\left(\mathcal{E}_{0}\right)},
\end{aligned}
$$

where $\mathcal{E}_{0}$ is the ensemble prepared in our demonstration.

\section{APPENDIX B: EXPERIMENTAL DETAILS}

\section{Preparation of the 12 MUB qutrit states}

We use a beamlike type-II spontaneous parametric downconversion from a BBO crystal to generate photon pairs $H V$, where $H$ and $V$ denote the horizontal and vertical polarization, respectively. One photon of polarization $V$ from the photon pair is created for the heralded trigger, and the other photon of polarization $H$ is applied to prepare the qutrit states in Eq. (A1). The part of state preparation is performed by a DPBS (double polarization beam splitter), a small-angle prism that adjusts the phase between the paths $(0,1)$ and path $(2)$, two half-wave plates, and a quarter-wave plate. We can acquire all

TABLE VII. Projectors angles: $C$ and $D$ bases with $\left(r_{x}, r_{y}\right)=$ $(1 / 2,1)$.

\begin{tabular}{lccccc}
\hline \hline Projector & Phase shifter & QWP & HWP3 & QWP & HWP5 \\
\hline$J_{1,1}$ & 258.8 & 37.4 & 71 & 52 & 26 \\
$J_{1,2}$ & 120 & 135 & 82.5 & 60.7 & 30.35 \\
$J_{1,3}$ & 251.2 & 37.4 & 26 & 52 & 26 \\
$J_{2,1}$ & 300 & 135 & 52.5 & 60.7 & 30.35 \\
$J_{2,2}$ & 168.8 & 142.6 & 78.6 & 52 & 26 \\
$J_{2,3}$ & 251.2 & 52.6 & 19 & 52 & 26 \\
$J_{3,1}$ & 30 & 90 & 19.2 & 52 & 26 \\
$J_{3,2}$ & 30 & 180 & 25.8 & 52 & 26 \\
$J_{3,3}$ & 210 & 45 & 22.5 & 60.7 & 30.35 \\
\hline \hline
\end{tabular}

TABLE VIII. Prior measurements: detector counts with various inputs.

\begin{tabular}{lrrrr}
\hline \hline & Detector 1 & Detector 2 & Detector 3 & Possibility \\
\hline$A 1$ & 51480 & 86 & 1318 & 0.973451 \\
$A 2$ & 51630 & 114 & 748 & 0.983578 \\
$A 3$ & 49430 & 72 & 30 & 0.997941 \\
$B 1$ & 49470 & 330 & 714 & 0.979332 \\
$B 2$ & 52100 & 926 & 704 & 0.969663 \\
$B 3$ & 41140 & 226 & 636 & 0.979477 \\
$C 1$ & 39130 & 554 & 498 & 0.973819 \\
$C 2$ & 49620 & 272 & 468 & 0.985306 \\
$C 3$ & 50910 & 340 & 532 & 0.983160 \\
$D 1$ & 52840 & 1182 & 872 & 0.962582 \\
$D 2$ & 53110 & 928 & 1048 & 0.964129 \\
$D 3$ & 51510 & 278 & 976 & 0.976234 \\
\hline \hline
\end{tabular}

12 MUB qutrit states when we set different plate angles and different phase shifts due to different optical-path lengths of the $H$ component and the $V$ component in the prism. Detailed angles of all involved wave plates for the three remaining MUBs $(B, C, D)$ are listed in Table II.

For instance, the HWP2 at $-60^{\circ}$ transforms the state $\frac{1}{\sqrt{3}}(|0\rangle+|1\rangle+|2\rangle)$ into $\frac{1}{\sqrt{3}}\left(e^{i \frac{2}{3} \pi}|0\rangle+e^{i \frac{2}{3} \pi}|1\rangle+|2\rangle\right)$. Then with the first QWP rotated at $45^{\circ}$, the state is converted into $\frac{1}{\sqrt{3}}\left(e^{i \frac{2}{3} \pi}|0\rangle+e^{-i \frac{2}{3} \pi}|1\rangle+|2\rangle\right)$, which is the state $\left|\psi_{2}^{B}\right\rangle$ in Eq. (A1).

We test the fidelity of these eigenstates in Table III, and the average fidelity of these prepared states is $F=0.977$ in our experiment.

\section{Incompatible and compatible measurements}

For obtaining the quantum incompatible witness, we perform the post measurement and prior measurement. Aiming at different measurement bases for different ensembles, we calculate the measurement settings, and the detailed angles of phase shifter, HWP3, HWP5, and two QWPs are adjusted before the photon entered the four $\mathrm{Si}$ single photon detectors.

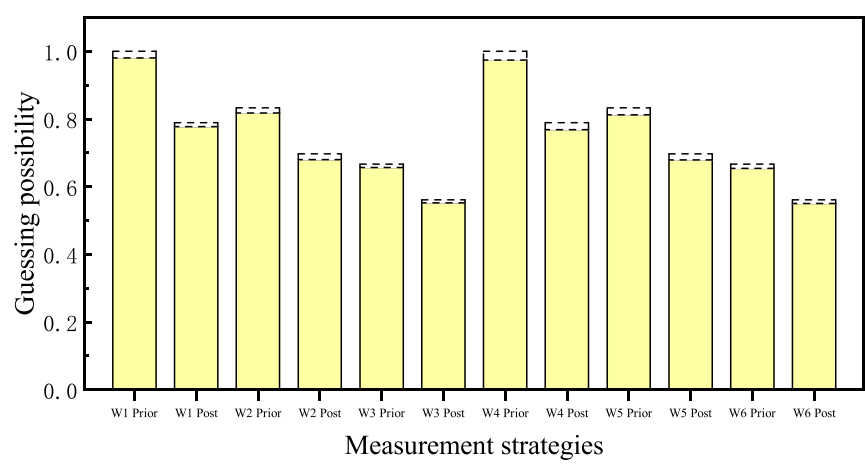

FIG. 5. Experimental and theoretical guessing probabilities with two strategies. Experimental results of the guessing possibility from six ensembles are shown in yellow bars and the dash empty bars indicate ideal measurement results for comparison. 
In the strategy with the post-measurement information, the guesser uses the measurements $J$ defined in the above section, which are implemented via modifying the angles shown in Tables IV and V for $A$ and $B$ bases and Tables VI and VII for $C$ and $D$ bases.

In the other strategy with the prior measurement information, the guesser has access to prior information of $X$ and $Y$.
Then he can choose a measurement $M$ for the states from $\mathcal{E}_{X}$ and a measurement $N$ for $\mathcal{E}_{Y}$ in Eq. (6). The results are shown in Table VIII.

The guessing probabilities in six ensembles with two different strategies are shown in Fig. 2. Figure 5 shows the theoretical and experimental guessing probabilities.
[1] H. P. Robertson, The uncertainty principle, Phys. Rev. 34, 163 (1929).

[2] T. Heinosaari, T. Miyadera, and M. Ziman, An invitation to quantum incompatibility, J. Phys. A: Math. Theor. 49, 123001 (2016).

[3] N. Brunner, D. Cavalcanti, S. Pironio, V. Scarani, and S. Wehner, Bell nonlocality, Rev. Mod. Phys. 86, 419 (2014).

[4] M. T. Quintino, T. Vértesi, and N. Brunner, Joint Measurability, Einstein-Podolsky-Rosen Steering, and Bell Nonlocality, Phys. Rev. Lett. 113, 160402 (2014).

[5] R. Uola, T. Moroder, and O. Gühne, Joint Measurability of Generalized Measurements Implies Classicality, Phys. Rev. Lett. 113, 160403 (2014).

[6] S.-L. Chen, C. Budroni, Y.-C. Liang, and Y.-N. Chen, Natural Framework for Device-Independent Quantification of Quantum Steerability, Measurement Incompatibility, and Self-Testing, Phys. Rev. Lett. 116, 240401 (2016).

[7] Y.-C. Liang, R. W. Spekkens, and H. M. Wiseman, Specker's parable of the overprotective seer: A road to contextuality, nonlocality and complementarity, Phys. Rep. 506, 1 (2011).

[8] B. Amaral and M. T. Cunha, On Graph Approaches to Contextuality and their Role in Quantum Theory (Springer, Berlin, 2018).

[9] Z.-P. Xu and A. Cabello, Necessary and sufficient condition for contextuality from incompatibility, Phys. Rev. A 99, 020103(R) (2019).

[10] M. Um, Q. Zhao, J. Zhang, P. Wang, Y. Wang, M. Qiao, H. Zhou, X. Ma, and K. Kim, Randomness Expansion Secured by Quantum Contextuality, Phys. Rev. Appl. 13, 034077 (2020).

[11] N. Gisin, G. Ribordy, W. Tittel, and H. Zbinden, Quantum cryptography, Rev. Mod. Phys. 74, 145 (2002).

[12] A. Fine, Hidden Variables, Joint Probability, and the Bell Inequalities, Phys. Rev. Lett. 48, 291 (1982).

[13] M. M. Wolf, D. Perez-Garcia, and C. Fernandez, Measurements Incompatible in Quantum Theory Cannot be Measured Jointly in Any Other No-Signaling Theory, Phys. Rev. Lett. 103, 230402 (2009).

[14] C. Carmeli, T. Heinosaari, and A. Toigo, Quantum Incompatibility Witnesses, Phys. Rev. Lett. 122, 130402 (2019).

[15] P. Skrzypczyk, I. Šupić, and D. Cavalcanti, All Sets of Incompatible Measurements Give an Advantage in Quantum State Discrimination, Phys. Rev. Lett. 122, 130403 (2019).

[16] R. Uola, T. Kraft, J. Shang, X.-D. Yu, and O. Gühne, Quantifying Quantum Resources with Conic Programming, Phys. Rev. Lett. 122, 130404 (2019).

[17] O. Guhne and G. Toth, Entanglement detection, Phys. Rep. 474, 1 (2009).

[18] R. Horodecki, P. Horodecki, M. Horodecki, and K. Horodecki, Quantum entanglement, Rev. Mod. Phys. 81, 865 (2009).
[19] M. Ringbauer, T. R. Bromley, M. Cianciaruso, L. Lami, W. Y. S. Lau, G. Adesso, A. G. White, A. Fedrizzi, and M. Piani, Certification and Quantification of Multilevel Quantum Coherence, Phys. Rev. X 8, 041007 (2018).

[20] Y.-Q. Nie, H. Zhou, J.-Y. Guan, Q. Zhang, X. Ma, J. Zhang, and J.-W. Pan, Quantum Coherence Witness with Untrusted Measurement Devices, Phys. Rev. Lett. 123, 090502 (2019).

[21] X. Zhang, Y. Liu, and X. Yuan, Quantifying coherence with untrusted devices, arXiv:1812.00844.

[22] R. Uola, C. Budroni, O. Gühne, and J.-P. Pellonpää, One-to-one Mapping between Steering and Joint Measurability Problems, Phys. Rev. Lett. 115, 230402 (2015).

[23] P. Skrzypczyk and N. Linden, Robustness of Measurement, Discrimination Games, and Accessible Information, Phys. Rev. Lett. 122, 140403 (2019).

[24] J. Bae, D. Chruściński, and M. Piani, More Entanglement Implies Higher Performance in Channel Discrimination Tasks, Phys. Rev. Lett. 122, 140404 (2019).

[25] G. Styliaris and P. Zanardi, Quantifying the Incompatibility of Quantum Measurements Relative to a Basis, Phys. Rev. Lett. 123, 070401 (2019)

[26] W. K. Wootters and B. D. Fields, Optimal state-determination by mutually unbiased measurements, Ann. Phys. (NY) 191, 363 (1989).

[27] I. D. Ivonovic, Geometrical description of quantal state determination, J. Phys. A: Math. Gen. 14, 3241 (1981).

[28] D. Giovannini, J. Romero, J. Leach, A. Dudley, A. Forbes, and M. J. Padgett, Characterization of High-Dimensional Entangled Systems via Mutually Unbiased Measurements, Phys. Rev. Lett. 110, 143601 (2013).

[29] R. B. A. Adamson and A. M. Steinberg, Improving Quantum State Estimation with Mutually Unbiased Bases, Phys. Rev. Lett. 105, 030406 (2010).

[30] I.-C. Yu, F.-L. Lin, and C.-Y. Huang, Quantum secret sharing with multilevel mutually (un)biased bases, Phys. Rev. A 78, 012344 (2008).

[31] N. J. Cerf, M. Bourennane, A. Karlsson, and N. Gisin, Security of Quantum Key Distribution Using $d$-Level Systems, Phys. Rev. Lett. 88, 127902 (2002).

[32] C. Carmeli, T. Heinosaari, T. Miyadera, and A. Toigo, Witnessing incompatibility of quantum channels, J. Math. Phys. 60, 122202 (2019).

[33] D. Rosset, F. Buscemi, and Y.-C. Liang, Resource Theory of Quantum Memories and Their Faithful Verification with Minimal Assumptions, Phys. Rev. X 8, 021033 (2018).

[34] X. Yuan, Y. Liu, Q. Zhao, B. Regula, J. Thompson, and M. Gu, Robustness of quantum memories: An operational resourcetheoretic approach, arXiv:1907.02521. 